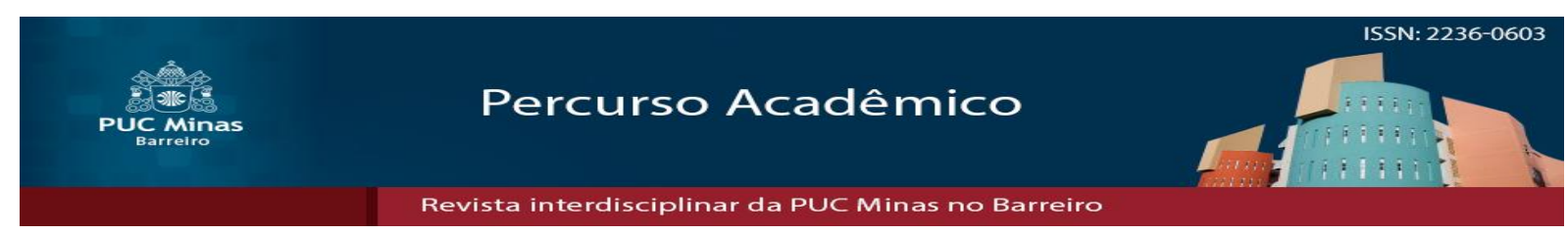

\title{
Avanços da lobectomia por cirurgia torácica vídeo assistida no tratamento do câncer de pulmão
}

\section{Advances of video-assisted thoracic surgery lobectomy in lung cancer treatment}

\author{
Frederico Luiz Pinto Coelho ${ }^{1}$ \\ Roberta Pereira de Miranda Franco ${ }^{2}$

\section{RESUMO}

Introdução: $O$ câncer de pulmão é a principal causa de morte por câncer no Brasil e no mundo. Grande parte dos pacientes é diagnosticada em fases avançadas da doença, onde as opções de tratamento são paliativas. Apenas 20-30\% dos doentes com câncer de pulmão são diagnosticados precocemente e, para estes, o tratamento cirúrgico é o padrão ouro, considerado o único tratamento curativo em estágios iniciais. O advento da técnica de lobectomia por cirurgia torácica videoassistida (CTVA) trouxe novas perspectivas ao tratamento do câncer de pulmão, iniciando uma nova era na cirurgia torácica. Objetivo: Descrever os avanços da técnica de CTVA no tratamento do câncer de pulmão, compreendendo potenciais vantagens e desvantagens em relação à lobectomia convencional. Materiais e métodos: Este estudo foi construído por meio de revisão bibliográfica de textos referência no assunto e artigos indexados nas bases PubMed e Scielo. Discussão: A toracotomia aberta provoca um grande trauma com ampla resposta imunológica, aumentando a morbimortalidade pós-operatória. Com o advento da cirurgia laparoscópica, chegamos à Cirurgia Torácica Vídeoassistida, suprimindo toracotomias abertas e suas consequências. A lobectomia por CTVA agora é comumente usada com resultados superiores, utilizando menos portas de acesso e sem a necessidade de afastar costelas ou seccionar músculos, o que propicia aos pacientes uma recuperação mais rápida. Entre os benefícios documentados da CTVA está a realização de incisões menores, com menor perda de sangue, menor risco de infecção, menor intensidade da dor, menor índice de complicações ventilatórias, menor liberação de mediadores inflamatórios, entre outros. Grandes massas tumorais, invasões extensas e certas complicações cirúrgicas perdem o benefício da abordagem minimamente invasiva do tórax. Conclusão: Apesar da gravidade, o câncer de pulmão tem cura quando identificado precocemente, e o seu tratamento pode ser feito por meio de cirurgia minimamente invasiva, sem prejuízo no resultado oncológico dos pacientes.

Palavras-chave: Câncer de pulmão. Lobectomia. Cirurgia torácica. Artigo científico. Cirurgia minimamente invasiva.

\begin{abstract}
Foreword: Lung cancer is the leading cause of cancer death in Brazil and worldwide. Most patients are diagnosed at advanced stages of the disease, where treatment options are palliative. Only 20-30\% of lung cancer patients are diagnosed early, and for these, surgical treatment is the gold standard, considered the only curative treatment in the early stages. The advent of the video-assisted thoracic surgery (VATS) lobectomy technique has brought new perspectives to the treatment of lung cancer, ushering in a new era in thoracic surgery. Objective: To describe the advances in the technique of VATS in the treatment of lung cancer, including potential advantages and disadvantages compared to conventional lobectomy. Materials and methods: This study was built by literature review of reference texts on the subject and articles indexed in PubMed and Scielo databases. Discussion: Open thoracotomy causes major trauma with a broad immune response, increasing postoperative morbidity and mortality. With the advent of laparoscopic surgery, we came to Video-assisted Thoracic Surgery, suppressing open thoracotomies and
\end{abstract}

\footnotetext{
Artigo recebido em 25 de outubro de 2019 e aprovado em 20 dezembro de 2019

${ }^{1}$ Acadêmico do 11 período de medicina da PUC Minas - Betim, Brasil. E-mail: fredlpc@yahoo.com.br

${ }^{2}$ Acadêmico do 11으 período de medicina da PUC Minas - Betim. Brasil. E-mail: roberta017@hotmail.com
} 
their consequences. VATS lobectomy is now commonly used with superior results, utilizing fewer access doors and without the need to dislocate ribs or sever muscles, which provides patients with faster recovery. Documented benefits of VATS include smaller incisions, lower blood loss, lower risk of infection, lower pain intensity, lower rate of ventilatory complications, lower release of inflammatory mediators, among others. Large tumor masses, extensive invasions, and certain surgical complications lose the benefit of the minimally invasive chest approach. Conclusion: Despite its severity, lung cancer is curable when identified early, and its treatment can be done by minimally invasive surgery, without affecting the cancer outcome of patients

Key-words: Lung cancer. Lobectomy Thoracic surgery. Scientific article. Minimally invasive surgery.

\section{INTRODUÇÃO}

O câncer de pulmão é a doença maligna mais comum em todo o mundo. Os dados do INCA de 2018 apontam que, de todos os novos casos de câncer, 13\% são de câncer de pulmão. Destaca-se também que o câncer de pulmão é o que mais mata no mundo, sendo responsável por 20-30\% de todas as mortes por câncer. 10\% dos casos de Câncer de Pulmão são causados por predisposição genética e por risco ocupacional (exposição a substâncias químicas, hidrocarbonetos aromáticos, arsênico, níquel, asbestos, agrotóxicos etc.). Os outros $90 \%$ são causados pelo cigarro. Afinal, dados apontam que $90 \%$ dos pacientes com câncer de pulmão são fumantes e que esses $90 \%$ de tumores poderiam ser evitados simplesmente parando de fumar. É comprovado que fumantes têm 20 vezes mais chance de desenvolverem CA de Pulmão, já que a fumaça do cigarro tem mais de 4.700 substâncias tóxicas. Só o alcatrão, por exemplo, é composto de mais de 40 substâncias cancerígenas. (Entre outras substâncias nocivas temos a nicotina, a acroleína, o benzeno, o tolueno, o arsênico, níquel acetaldeído, naftalina, formaldeído, amônia, entre outros). O cigarro não é mais considerado apenas um fator de risco para o câncer de pulmão, mas sim o próprio agente etiológico. Sua fumaça causa, ao longo do tempo, metaplasia, displasia, carcinoma in situ e carcinoma invasivo.

O Câncer de pulmão tem então alta incidência, alta mortalidade, alta letalidade. É um câncer altamente agressivo, pois uma vez diagnosticado, gera uma sobrevida curta ao paciente. Após o diagnóstico a expectativa de vida é de 7 meses a 5 anos, sendo a taxa de sobrevida estimada em 5 anos entre $10 \%$ a $20 \%$, gerando uma taxa de $80 \%$ de mortalidade. Segundo a Organização Mundial de Saúde (OMS), cerca de 2.800.000 pessoas morreram por câncer de pulmão em 2017. O câncer de pulmão segue de forma assintomática, o faz com que a maioria dos diagnósticos ocorra em estágios avançados da doença (Estágios III e IV), onde restam apenas tratamentos paliativos como a 
quimioterapia e a radioterapia. Porém, quando detectado precocemente (nos estágios I e II) é passível de cirurgia, o único tratamento curativo possível. Os sintomas surgem após determinado tempo e dependem do tamanho e da localização do tumor. Pode ocorrer tosse devido à irritação local, hemoptise devido à ulceração tumoral e/ou dispneia, pela obstrução. Em alguns países é recomendado rastreamento anual para grupos populacionais de alto risco: pacientes com idade entre 55 a 80 anos, com carga tabágica superior a 30 anos/maço e fumantes com boa saúde em geral, sem esperar o aparecimento dos sintomas. Assim sendo, o diagnóstico é feito por meio dos exames de Tomografia Computadorizada com baixa dose de radiação, sem contraste, e/ou biópsia pulmonar broncoscópica ou transtorácica.

Entre os procedimentos cirúrgicos consensuais podemos citar a pneumectomia, a lobectomia e a bi-lobectomia, que podem ser acompanhadas de broncoplastias, carinectomia, ressecção de parede torácica, retirada de linfonodos, entre outras medidas. As ressecções menores em que a lobectomia não é consensual devem ser evitadas, pois estão associadas a um aumento no índice de recidiva local, interferindo na sobrevida desses doentes. Há cerca de 60 anos, a pneumectomia era a opção cirúrgica disponível para a ressecção completa de tumores pulmonares. Porém, a toracotomia aberta provoca um grande trauma com ampla resposta imunológica, aumentando a morbidade e mortalidade pós-operatórias. Atualmente, a lobectomia tornou-se a melhor opção, com menor morbidade e resultados em longo prazo superponíveis. São notórios os avanços da técnica de CTVA no tratamento do câncer de pulmão, compreendendo potenciais vantagens e desvantagens em relação à lobectomia convencional.

A lobectomia por Cirurgia Torácica Videoassistida (CTVA) agora é comumente usada com resultados superiores. Muitos autores consideram a toracotomia com secção muscular e uso de afastador com lesão periostial dos arcos costais, músculos e nervos torácicos, uma patologia a mais na intenção do tratamento da doença que gerou a intervenção.

\section{DESENVOLVIMENTO}

O Câncer de pulmão tem alta incidência, alta mortalidade, e elevadas taxas de letalidade. Considerado um câncer altamente agressivo, com sobrevida curta após ter sido feito seu diagnóstico, é necessário estabelecer os critérios cirúrgicos que permitam uma melhor recuperação pós-operatória e com melhor prognóstico para paciente. 
Ainda que não haja dados exatos a respeito do número de procedimentos cirúrgicos realizados para tratar pacientes com câncer de pulmão, segundo o Departamento de Informática do SUS — um banco de dados do sistema público de saúde, que cobre aproximadamente $75 \%$ da população brasileira — uma mediana de 964 lobectomias pulmonares foi realizada anualmente entre os anos de 2007 a 2014. Embora seja uma técnica já bem estabelecida mundialmente, a Cirurgia Torácica Videoassistida (CTVA) ainda não faz parte da rotina de muitos centros de cirurgia torácica no Brasil.

É válido ressaltar que o câncer de pulmão pode ser prevenido com a cessação do tabagismo (principal fator de risco para a patologia) e que pode ser detectado precocemente com medidas de rastreamento, pois, apesar da gravidade, o câncer de pulmão tem cura quando identificado de forma precoce, permitindo ao paciente possibilidades maiores de sobrevida quanto mais inicialmente for detectado o tumor.

\section{METODOLOGIA}

Pesquisa em banco de dados: PubMed, Lilacs, Scielo, OMS, Ministério da Saúde, INCA, através de revisão bibliográfica da literatura, a partir do ano de 2002.

\section{RESULTADOS}

Observa-se que a lobectomia por Cirurgia Torácica Videoassistida (CTVA) apresentou resultados notadamente superiores comparados à cirurgia aberta: incisões menores (minimamente invasivas), consequentemente menor perda de sangue e menor risco de infecção intra e pós-operatória. Não possui a necessidade de seccionar ou de afastar as costelas, apresentando assim um menor índice de complicações ventilatórias, com menor liberação de mediadores inflamatórios. O tempo cirúrgico também foi menor, assim como o custo do procedimento cirúrgico para o hospital. A imagem gerada no visor é ampliada em alta definição, o que gera maior segurança para toda a equipe envolvida no processo cirúrgico. Além das vantagens cirúrgicas, o procedimento minimamente invasivo permite que pacientes idosos, os quais talvez não estariam aptos a uma cirurgia aberta e às suas repercussões intraoperatórias e póscirúrgicas, tenham a possibilidade de serem operados. A intensidade da dor no pósoperatório também é menor e o resultado estético melhor, com cicatrizes reduzidas. Também observamos menor tempo de drenagem, menor tempo de internação e um retorno mais precoce às atividades diárias. 


\section{DISCUSSÃO}

A Cirurgia Torácica Videoassistida (CTVA) pertence a um novo paradigma, um novo conceito na Cirurgia Torácica: a cirurgia torácica minimamente invasiva (CTMI), procedimento totalmente orientado por videotoracoscopia, sem a utilização de afastador de costelas, com pequenas incisões de trabalho, utilizando menos portas de acesso, propiciando aos pacientes uma recuperação mais rápida e um retorno precoce às atividades do dia a dia. Tudo isto com o mesmo rigor cirúrgico e rigor oncológico da toracotomia aberta.

A lobectomia por CTVA só é contraindicada em situações de grandes massas tumorais, invasões extensas ou metástases. Além disto, certas complicações cirúrgicas perdem o benefício da abordagem minimamente invasiva do tórax necessitando de conversão para cirurgia aberta para corrigir o problema. Por isso, entre os princípios da CTVA está a necessidade de se ter mesa auxiliar preparada para uma possível conversão para a cirurgia convencional. A cirurgia é feita então colocando-se o paciente em decúbito lateral e imobilizando-o. Em seguida realiza-se a anestesia geral e a intubação, que deve ser feita com tubo seletivo monopulmonar (tubo de Carlens), isolando o pulmão a ser operado. São então realizadas 4 incisões ou portais: um portal de $10 \mathrm{~mm}$ para a ótica, um portal de $10 \mathrm{~mm}$ para trocater da mão direita do cirurgião, um portal de $0,5 \mathrm{~cm}$ para o trocater da mão esquerda e uma incisão para o cirurgião auxiliar. A lobectomia é realizada com a dissecção da veia pulmonar do respectivo lobo com preservação da veia para os demais lobos. Em seguida realiza-se a dissecção do tronco arterial para o respectivo lobo. Adiante, é preciso se identificar ramo arterial recorrente e dissecá-lo. Por conseguinte, realiza-se a dissecação do brônquio para o lobo respectivo e por último realiza-se a dissecção e a remoção dos linfonodos próximos.

\section{CONSIDERAÇÕES FINAIS}

A CTVA segue em pleno avanço de suas técnicas e cada vez mais revoluciona a forma como os cirurgiões tratam a patologia pulmonar, iniciando uma nova era de progresso e de desenvolvimento na cirurgia torácica. Com resultados notadamente superiores por meio da Cirurgia Torácica Minimamente Invasiva (CTMI), pacientes diagnosticados com câncer de pulmão e que tenham possibilidade cirúrgica, poderão se beneficiar de um procedimento com menores riscos intra-operatórios, além de uma 
recuperação mais rápida, que lhes permita retorno precoce às suas atividades de vida diária.

\section{REFERÊNCIAS}

ARAUJO, Luiz Henrique et al. Lung cancer in Brazil. J. Bras. Pneumol., São Paulo, v. 44, n.1, p. 55-64, fev. 2018.

BARBOSA, Felipe de Galiza. PET/TC na avaliação de nódulo pulmonar solitário. Radiol. Bras., São Paulo, v .49, n. 2, p. XI, abr. 2016.

BRASIL. Ministério da Saúde. Diretrizes diagnósticas e terapêuticas em oncologia carcinoma de pulmão. Portaria No 957, de 26 de setembro de 2014.

BRASIL. Ministério da Saúde. Estimativa 2018: Incidência de Câncer no Brasil. Instituto Nacional do Câncer. Rio de Janeiro, 2017.

CARVALHO, Erlon de Ávila et al. Aspectos táticos e técnicos observados nas ressecções pulmonares anatômicas por videotoracoscopia. Revista Médica de Minas Gerais. Minas Gerais, v. 27, p.52-57, 2017.

FORTE, Vicente. Fatores de risco nas lobectomias realizadas para tratar o carcinoma brônquico. J. bras. pneumol., São Paulo, v. 32, n. 6, p. 30-32, fev. 2006.

GIACOMELLI, Iron Pedro et al. Câncer de pulmão: dados de três anos do registro hospitalar de câncer de um hospital do sul do Brasil. Arquivos Catarinenses de Medicina, v.46, n.3, p. 129-146, set. 2017.

GUIMARÃES, Anderson Nassar et al. Cirurgia Torácica Minimamente Invasiva Ressecções pulmonares Cirurgia Torácica Vídeo Assistida (CTVA). Pulmão RJ. Rio de Janeiro, v. 23, n.01, p. 16-19, 2014.

HAMERSCHLAK, Nelson et al. Câncer de pulmão: prevenção e cura. Educ. Contin. Saúde Einstein. São Paulo, v.10, n. 03, p. 130-134, 2012.

HOCHHEGGER, Bruno et al. PET/TC em câncer de pulmão: indicações e achados. J. Bras. Pneumol., São Paulo, v. 41, n. 3, p.264-274, jul. 2015.

ISMAEL, Gustavo Fernando V. Aspectos clínicos e histopatológicos em câncer de pulmão: análise dos dados de uma instituição no interior paulista entre 1997 e 2008. Revista Brasileira de Oncologia Clínica. São Paulo, v. 7, n. 22, p. 73-78, dez. 2010.

LADRON DE GUEVARA H, DAVID; PEFAUR D,RAÚL. PET/CT em câncer pulmonar. Rev.méd.Chile, Santiago, v. 138, n. 11, p. 1441-1450, nov. 2010.

OLIVEIRA, Christopher; SARAIVA, António. Estudo comparativo do diagnóstico de câncer pulmonar entre tomografia computadorizada e broncoscopia. Radiol. Bras., São Paulo, v. 43, n. 4, p. 229-235, ago, 2010. 
Programa de auto-avaliação em cirurgia. Câncer de pulmão. Colégio Brasileiro de Cirurgiões. Ano 3, Fascículo II, ago.2004.

RODRIGUES, Wellington Pereira et al. Câncer de pulmão e suas consequências na qualidade de vida. Revista Saúde em Foco. Teresina/Piauí, ed.10, p.101-110, 2018.

SCHNEIDER, Airton et al. Estudo comparativo entre lobectomia e segmentectomia estendida para o tratamento do carcinoma brônquico não de pequenas células em estágios iniciais. J. bras. pneumol., São Paulo, v. 30, n. 5, p. 433-438, out. 2004.

SILVA, José Alencar Gomes. Estimativa 2018: incidência de câncer no Brasil / Instituto Nacional de Câncer. Coordenação de Prevenção e Vigilância, MS. Rio de Janeiro, 2017.

SODER, Stephan Adamour et al. Ressecções pulmonares anatômicas por cirurgia torácica videoassistida: experiência de 117 casos em um centro de referência no Brasil. J Bras. Pneumol., São Paulo, v. 43, n. 2, p. 129-133, jan. 2016.

TERRA, Ricardo Mingarini et al. Lobectomia pulmonar robótica para tratamento do câncer de pulmão e de metástases pulmonares: implantação do programa e experiência inicial. J. Bras. Pneumol., São Paulo, v. 42, n. 3, p. 185-190, fev. 2016.

TSUKAZAN, Maria Teresa Ruiz et al. Câncer de pulmão: mudanças na histologia, sexo e idade nos últimos 30 anos no Brasil. J. Bras. Pneumol., São Paulo, v. 43, n. 5, p. 363-367, out. 2017.

ZAMBONI, Mauro. Epidemiologia do câncer do pulmão. J. bras. pneumol., São Paulo, v. 28, n. 1, p. 41-47, fev. 2002. 